Intractable infantile spasms are associated with reduction in facial expression of positive affect and impaired use of positive emotion during social communication. (Caplan R, Guthrie D, Komo S, Shields WD, Sigman M. Infantile spasms: Facial expression of affect before and after epilepsy surgery. Brain Cogn March 1999;39:116-132). (Reprints: Rochelle Caplan, Department of Psychiatry and Behavioral Sciences, UCLA, 760 Westwood Plaza, Los Angeles, CA 90024).

COMMENT. In addition to the well known association of autistic behavior with infantile spasms, children with intractable spasms have a reduction in positive affect during social communication. The use of positive affect may be increased following epilepsy surgery, but the relation of this effect to the localization of brain pathology and functional plasticity of facial expression of affect requires further study.

The origin of hypsarrhythmia and tonic spasms in West syndrome is discussed in relation to the report of a 3-year-old girl with porencephaly and hydrocephalus with focal hypsarrhythmia from Tohoku University, Sendai, Japan (Haginoya K et al. Brain Dev March 1999;21:129-131). The left memisphere was completely defective, and hypsarrhythmia was seen over the residual right frontal cortex. Despite focal EEG findings, tonic spasms were symmetrical, and an intact brainstem appeared to be essential for the occurrence of spasms. An ictal SPECT showed hyperperfusion of the brainstem and cerebellum.

\title{
SUBPIAL RESECTION FOR LANDAU-KLEFFNER SYNDROME
}

Speech and language outcome of 14 children treated for Landau-Kleffner syndrome by multiple subpial transection was evaluated at Rush-Prebyterian-St Luke's Medical Center, Chicago. Language deficits presented at a mean age of 4 years, with a range of 3.0-6.5 yrs, and previous history of language and cognitive development was normal. The average age at time of surgery was 7.4 years, and the range was 5 to 13 years. Two patients had surgical complications; one developed meningitis and another suffered a stroke. Eleven (79\%) had significant postoperative improvement in receptive and expressive vocabulary. The extent of improvement was inversely correlated with age of onset and age of surgery, and directly correlated with the time elapsed between surgery and the time of the most recent postoperative language evaluation. A control group was not available. (Grote CL. Van Slyke P, Hoeppner J-AB. Language outcome following multiple subpial transection for Landau-Kleffner syndrome. Brain March 1999;122:561566). (Respond: Christopher Grote, Rush-Presbyterian-St Luke's Medical Center, 1653 W Congress Parkway, Chicago, IL 60612).

COMMENT. Landau-Kleffner syndrome is an acquired epileptic aphasia that develops in previously normal young children who lose previously acquired speech and language abilities. All patients selected for the study met these diagnostic criteria. The best predictor of language outcome following subpial resection was the amount of time elapsed between surgery and the time of language evaluation. In the absence of a control group, could these results be a function of the natural history of the disease? The following reports suggest that, although the Rush-Presbyterian experience is encouraging, controls may be needed to accurately evaluate the benefits of subpial resection.

Deonna $\mathrm{T}$ and associates of Lausanne, Switzerland, have reported an adult follow-up study of 7 patients with acquired epileptic aphasia beginning in childhood (Neuropediatrics 1989;20:132). Four showed no improvement, two were 
partially improved, and one man had made a complete recovery of speech and language. These authors subsequently reported a patient followed from age 3 to 18 years whose language and behavior correlated with abnormalities on the EEG. Improvement coincided with the disappearance of continuous spike wave during sleep and the onset of a unilateral focus (Roulet E, Deonna T et al. Epilepsia 1991;32:495). Paquier PF et al, Rotterdam, The Netherlands, in a follow-up of 6 patients for periods ranging from 3 to 19 years found the outcome of aphasia variable, with slow improvement in 4 , rapid recovery in 1 , and fluctuating course in 1. (Arch Neurol 1992;49:354). (see Progress in Pediatric Neurology I, PNB Publishers, 1991;pp217-218; and Vol II, 1994;pp57-58, for several references and commentary regarding outcome of L-K syndrome).

Landau-Kleffner syndrome (LKS) and electrical status epilepticus during sleep (ESES). Eleven patients with LKS and bitemporal ESES were followed for a mean of 9 yrs and 8 mos at the Neurological Institute, University of Bologna, Italy. Complete recovery of language and cognitive abilities occurred in $18 \%$, and $64 \%$ were mentally retarded. Poor outcome showed some relation to onset of aphasia before 4 years, duration for longer than 1 year, and long-lasting ESES, probably preexisting speech delay. The common origin of LKS and ESES is confirmed by recent functional brain imaging studies. The elimination of ESES appears to be important in prognosis of LKS. (Rossi PG, Parmeggiani A, Posar A et al. Brain Dev March 1999;21:90-98).

\section{VALPROATE-INDUCED HYPERANDROGENISM IN GIRLS}

The reproductive endocrine function in 41 girls, 8 to 18 years old, treated with valproate for epilepsy and in 54 healthy control girls was evaluated at the University of Oulu, Finland. Mean serum testosterone concentrations of pubertal and pre- and post-pubertal girls taking valproate were significantly higher than in controls. Hyperandrogenism (serum testosterone levels 2SD above mean control levels) occurred in one third of prepubertal and pubertal valproate treated girls, and more than one-half of postpubertal girls were affected. Postpubertal girls taking valproate were more obese than controls, but the frequency of menstrual irregularities was not increased. (Vainionpaa LK, Rattya J, Knip M et al. Valproate-induced hyperandrogenism during pubertal maturation in girls with epilepsy. Ann Neurol April 1999;45:444-450). (Respond: Dr Vainionpaa, Department of Pediatrics, University of Oulu, FIN-90220 Oulu, Finland).

COMMENT. Monitoring of serum testosterone, height, weight, and Tanner staging of genitalia and pubic hair, during valproate therapy in adolescent girls with epilepsy may be indicated, particularly when antiepileptic medication is required for extended periods, as in juvenile absence and myoclonic epilepsies. In adolescent girls and especially in patients developing hyperandrogenism, a substitute therapy such as lamotrigine should be considered. Hyperandrogenism is a complication of valproate therapy during pubertal maturation of girls with epilepsy, and the frequency of this side effect increases in postpubertal patients.

The University of Oulu investigators have previously reported on the incidence of obesity, polycystic ovaries, and hyperandrogenism in women taking valproate. Fourteen (64\%) of 22 women receiving valproate were affected compared to $9(21 \%)$ of 43 on carbamazepine and $8(19 \%)$ of 43 untreated controls. Polycystic ovarian syndrome, hyperandrogenic chronic anovulation, is characterized by hirsutism and menstrual disorders. (see Progress in Pediatric Neurology II and III, PNB Publ, 1994 \& 1997 for further commentary on endocrine effects of valproate, including pubertal arrest). 